Review

\title{
Behaviors of Persons with Dementia: A Review and New Perspective for Future Research
}

Morgan J. Minyo ${ }^{1}$, Katherine S. Judge ${ }^{1,2, *}$

1. Department of Psychology, Cleveland State University, 2121 Euclid Ave, UN 244, Cleveland OH 44115, USA; E-Mails: m.j.watts@vikes.csuohio.edu; k.judge46@csuohio.edu

2. Center for Research and Education, Benjamin Rose Institute on Aging, Cleveland, $\mathrm{OH} 44120$, USA

* Correspondence: Katherine S. Judge; E-Mail: k.judge46@csuohio.edu

Academic Editor: Ladislav Volicer

Special Issue: Behavioral Symptoms of Dementia

\section{OBM Geriatrics}

2020, volume 4, issue 1

doi:10.21926/obm.geriatr.2001104
Received: July 30, 2019

Accepted: February 03, 2020

Published: February 07, 2020

\begin{abstract}
Through the assessment of previous and current literature on the behaviors experienced by persons with dementia, this review discusses the terminology, conceptual models, measurement techniques, and non-pharmacological interventions employed within behavioral research. Additionally, this review considers the illness experience and the need for more research using self-report methodology with persons with dementia in order to address the current gaps within the behaviour literature. With three main objectives, this review paper will address: (1) the literature on behaviors of persons with dementia; (2) the importance of and evidence of the illness experience of persons with dementia; and (3) future research goals and directions.
\end{abstract}

\section{Keywords}

Dementia; illness experience; person-centered care; behaviors; unmet needs

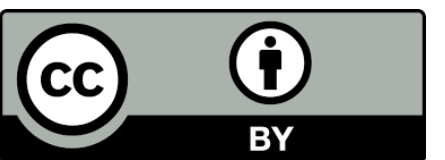

(c) 2020 by the author. This is an open access article distributed under the conditions of the Creative Commons by Attribution License, which permits unrestricted use, distribution, and reproduction in any medium or format, provided the original work is correctly cited. 


\section{Introduction}

Persons with dementia (PWDs) can experience a wide range of behaviors, such as wandering, agitation, and repetitive question asking. These behaviors can negatively impact PWD's quality of life and well-being [1]. Additionally, the expression and experience of these behaviors can negatively impact their informal family and friend caregivers and their formal, paid caregivers [1]. The following article provides an overview of the different behaviors experienced by PWDs and the significance of these behaviors for both PWDs and caregivers. As part of this, a review of different theoretical models used to study behaviors, current measurement techniques used to study behaviors in PWDs, and intervention programs aimed at reducing the impact of behaviors are included. This article is not intended to be a comprehensive and lengthy review of each of these important areas but rather an overview of the field and more importantly to identify gaps in the literature. Lastly, a discussion of future research directions is provided that hopefully will spur dialogue and next steps within the field. The article is written from the lens of the following overarching and interconnected concepts and include: person-centered care approach, understanding the illness experience, and the subjective experience of living with dementia.

The behavioral changes associated with dementia have long been studied due to the considerable danger, distress, and burden that these behaviors contribute to PWDs and caregivers [1]. Though the fifth edition of the Diagnostic and Statistical Manual of Mental Disorders (DSM V) defines dementia as a neurocognitive disorder, characterized most prominently by cognitive impairment within thinking and functional abilities [2], most caregivers are likely to identify behavioral and psychological symptoms of dementia (BPSD) as one of the most important features of the dementia illness due to the resulting caregiver burden and stress [3-5]. BPSD, also referred to within the literature as neuropsychiatric symptoms, behavioral problems, challenging behaviors or agitated behaviors, is defined as "signs and symptoms of disturbed perception, thought content, mood, or behavior" [6] and include a range of behaviors such as agitation, depression, apathy, repetitive questioning, psychosis, aggression, wandering, as well as various socially inappropriate behaviors [5]. More recently, researchers have begun discussing behaviors experienced by PWDs as expressions of unmet needs [7-9]. This is a rather unique perspective in viewing behaviors as it places behaviors as not a central symptom of a dementing illness but rather the result of complex social, emotional, and physical needs that are not being met due to the impact of cognitive impairment. Although individuals can experience behaviors at any point in their illness, research has found that as cognitive impairment worsens, behavioral symptoms increase in frequency [10].

Over the past 25 years, researchers have recognized the importance of BPSD in relation to various outcome measures as these symptoms are some of the most complex, stressful, and costly aspects of care $[2,5]$. The negative outcomes for PWDs associated with BPSD include increased risk of institutionalization and hospitalization [11-13], decreased functional abilities [14], and diminished aspects of quality of life [2]. Additionally, there are a myriad of negative health outcomes that caregivers are at risk for once these BPSD emerge and progress throughout the illness. For example, caregivers within the community are at an increased risk for caregiver psychiatric morbidity, increased prevalence of depression and anxiety, and caregiver burden [15]. BPSD also can have a negative impact on professional caregivers within nursing homes or facility settings including high levels of staff distress [16] and staff frustration with residents [17]. Whereas the specific prevalence rates for each type of possible BPSD varies across articles, for instance apathy in 27\% [18], 
aggression in 54\% [1], and agitation in 42\% of PWDs [1], many studies have identified that nearly all PWDs experience one or more BPSD at some point during their illness [19]. Recognizing the physical, mental, and emotional toll that BPSD can have on PWDs and caregivers, researchers have examined specific aspects of these behaviors in order to understand the underlying etiologies and related outcomes.

Within the literature, the term BPSD is a multi-dimensional construct with various classifications and conceptualizations. The description and context of these behaviors are important to consider in order to address BPSD within a research context and guide researchers' understanding of these behaviors. In a review by Desai and Grossberg [1], the classification of behavioral disturbances are discussed within three categories: (1) primary behavioral disturbances, which are caused by the underlying neurochemical changes associated with dementing illnesses (i.e. wandering, pacing, apathy, psychosis, depression); (2) secondary behavioral disturbances or those disturbances caused by co-morbid medical issues, pain, personal needs, or environmental factors; and (3) mixed behavioral disturbances, which are the result of primary and secondary disturbances that exacerbate the prevalence of one another.

Other researchers, such as Cohen-Mansfield and colleagues [20] conceptualize behaviors into positive behaviors (i.e. positive engagement with one's environment), problem behaviors (i.e. behaviors perceived as inappropriate and disruptive, such as agitation), and apathy (i.e. neither positive nor problem based behaviors). Additionally, these classifications can be used to further delineate behaviors, such as 'problem behaviors' can assess levels of: agitation, physically nonaggressive, physically aggressive, verbally aggressive, and/or verbally non-aggressive behaviors [21]. Other classifications of BPSD include Burgio's [4] description of behavioral disturbances as both behavioral excesses and deficits. Behavioral excesses include physical aggression, wandering, and disruptive vocalization, wherein the occurrence of the behavior(s) creates the disruptive problem for caregivers [15]. Whereas, behavioral deficits describe the non-occurrence of a behavior that results in a problem, such as the inability to self-dress, apathy, or social withdrawal [15].

Not only do the classification of behaviors differ within the literature, definitions of specific behaviors vary as well and have changed over time. Take for instance the behavior of agitation, which is one of the most extensively studied behaviors. Cohen-Mansfield and Werner [22] defined agitation as "socially inappropriate verbal or motor activity that is not a necessary by-product of a medical condition." Camp, Cohen-Mansfield, and Capezuti [23] conceptualized agitation as "resulting from an interaction among lifelong habits and personality, current physical and mental conditions, and environmental factors both physical and psychological." More recently, Volicer and colleagues [24] defined agitation as "an uninvoked symptom that communicates the experiencing of an unpleasant state of excitement." This range of definitions for agitation highlights the complex and multi-dimensional nature of BPSD. Though some BPSD receive heightened attention due to the intensive caretaking nature such as agitation, aggression, and wandering, other behaviors are of equal importance but are often overlooked. For instance, passive behaviors (i.e. apathy) characterized by a reduction in energy, drive, and initiative manifest in the majority of PWDs [25]. These behaviors are distinct from clinical depression and tend to increase in prevalence throughout the progression of the disease [26]. Additionally, passive behaviors have been associated with excessive disability, increased functional decline, and decreased quality of life [27], however, most of these behaviors go unattended as many caregivers focus on the more visible and attention garnering behaviors such as aggression and outward behaviors [28]. 
In consideration of the possible behaviors that could be experienced by PWDs as well as an attempt to alleviate BPSD, the literature provides a considerate amount of research on the distinct triggers and events proceeding BPSD and environments by which these behaviors have been observed. In a study conducted by Cohen-Mansfield and Werner [22], when an agitated behavior was presented, the authors noted who/what the behavior was directed at, the timing and location of the behavior, the physical environmental attributes, the social environment, and activities and stimulation. The authors observed several interesting findings. Agitated behaviors tended to decrease when participants were involved in structured activities whereas behaviors increased when participants were alone. Aggression increased when participants were engaged in social interactions and verbal agitation (i.e., screaming) tended to occur in residents' rooms. Other studies utilizing similar methods have found other informative outcomes. For example, when pain management efforts were utilized, agitation decreased [29]. Improving PWD's understanding of their caregiver's intentions before enacting personal care showed a reduction in their restiveness to care behaviors (often called aggressive behaviors) [30]. Lastly, increasing meaningful engagement in activities was associated with less wandering and reduced apathetic behaviors [24]. These studies highlight the triggering events and environmental contexts that improve or exacerbate BPSD and provide foundational support for the development of interventions that can alleviate BPSD and the resulting negative outcomes associated with BPSD.

Based on the literature, non-pharmacological approaches that are designed to address these needs have been recommended as the first line of intervention for the treatment of behavioral symptoms, with the exception of psychotic or dangerous symptoms in PWDs who have a previous history of mental health problems prior to the dementia diagnosis [31]. Pharmacological approaches typically utilize classes of psychotropic medication, such as benzodiazepines, anticonvulsants, and antidepressants for the treatment of aggression, psychotic symptoms, and/or agitation and have only shown modest effectiveness and for some individuals can result in negative and/or harmful side effects [32]. Non-pharmacological approaches, on the other hand, have demonstrated positive outcomes that encompass a vast array of behavioral, environmental, and caregiver supportive interventions [5]. Most non-pharmacological approaches are designed to address an underlying unmet need [1, 5, 22, 23, 33]. Through the plethora of research conducted on behaviors exhibited by PWDs, researchers have been able to identify, through observation and proxy-report techniques, various unmet needs that may be acting as triggers for BPSD. Taking a deeper look into how these non-pharmacological interventions for behaviors have been developed, the following sections will discuss several conceptual models, measurement techniques, as well as specific intervention protocols that have been utilized in this line of research.

\section{Conceptual Models}

Several conceptual models have been developed that provide a framework for understanding and studying behaviors of PWDs. Many of these conceptual models incorporate principles based on a person-centered care (PCC) approach [34]. The PCC approach emphasizes the 'individual' living with dementia and considers the complex and unique characteristics of individuals along with their social and physical environments. This approach offers a more comprehensive and holistic framework for understanding behaviors experienced by PWDs and, in contrast to a more traditional medical model approach, does not conceptualize dementia or the related behaviors as solely due 
to the underlying pathology of the illness [35]. By incorporating and utilizing a PCC approach, the conceptual models discussed below provide important information about the underlying root and key predictors of BPSD. Specifically, the following models are discussed and include a brief description and overview along with relevant research findings: the Need-driven Dementiacompromised Behavior Model, the Progressively Lowered Stress Threshold Model, the Unmet Needs Model, and the Stress Process Model for Caregivers and for Individuals with Dementia.

The first model discussed that incorporates a PCC approach is the Need-driven Dementiacompromised Behavior (NDB) Model. The NDB model examines behaviors by considering the purpose or meaning behind the behavior displayed by the PWD [7]. Previous terms for behaviors, such as 'disruptive' and 'challenging' express caregivers' viewpoints more than PWDs' perspectives. Though these behaviors may be challenging for caregivers, the NDB model suggests an alternative perspective by postulating that behaviors may actually be a response or an expression of a PWDs' needs [7]. Within this framework, the expression of a need reflects the background and proximal factors that can be found within the PWD, within the PWD's environment or perhaps both [7]. As such, background factors incorporate relatively stable constructs such as neurological, cognition, health status, and psychosocial factors that contribute to the expression of NDBs [7]. Whereas proximal factors have the ability to fluctuate and incorporate the current physical and social environment as well as the ever-changing needs of PWDs [7]. Examples of NDBs that may be observed due to both background and proximal factors include wandering, vocalizations, and physical aggression.

Norton, Allen, Snow, Hardin, and Burgio [36] tested this model by examining key background and proximal factors of PWDs as predictors of NDBs and their resistance to care in nursing homes. Results from this study found that the background characteristics of cognitive status and functional abilities were significant predictors of both memory-related NDBs and disruptive NDBs [36]. However, the significance for proximal factors, that included pain diagnoses and pain intensity, were not significant predictors of NDBs [36]. Specifically, individuals with more cognitive impairment displayed more memory-related behavior problems as well as disruptive behavior problems [36]. These finding are consistent with previous research that has found behavioral problems generally increase as a function of greater cognitive impairment [37].

Comparable to the NDB model, The Progressively Lowered Stress Threshold (PLST) [38] model focuses on intervening upon specific environmental stressors to decrease behaviors exhibited by PWDs [1]. The impetus for this model was based on the observation that many behaviors exhibited by PWDs were due to overwhelming levels of stress experienced by the PWD. As such, research was able to observe and document these behaviors along with the respective stress triggers. Of particular interest, researchers noted that as the disease advanced, PWDs had a lower threshold for triggers to elicit a stress response, demonstrating a progressive lowering of their stress threshold [39]. Based on these findings, the model provides caregivers with a method for organizing observations, making care decisions, and planning care that modifies stress inducing triggers and thus minimizing behaviors [39]. Research using PLST has found interesting findings demonstrating positive outcomes for caregivers, including reducing their reaction to behavioral symptoms [40, 41], lower appraisals of stress and burden [41], decreased feelings of uncertainty and unpredictability within the caregiving context [39], and increased quality of life for PWDs as reported by caregivers [40]. However, less research has been conducted on the effectiveness of using the PLST model to examine how PWDs subjectively experience their behavioral symptoms. 
The Unmet Needs Model [22] is another theoretical model that has been developed to understand the etiologies of behaviors of PWDs and has been used within a multitude of studies and interventions $[8,9,33,42,43]$. The Unmet Needs Model, similar to the NDBs model, states that the dementia process includes a decrease in the ability to meet one's own needs and provide for oneself due to the increasing difficulty with communication, physical abilities, and cognitive functioning [9]. According to this framework, behaviors may be a manifestation of an unmet need $[22,44]$ and these needs are a result of an imbalance in the relationship between lifelong habits, personality, current physical and mental states, and non-optimal environments [9]. These needs could include but are not limited to pain or physical discomfort, mental discomfort, social isolation, uncomfortable environmental conditions, or inadequate levels of stimulation [8].

Galindo- Garre, Volicer, and Van der Steen [42] applied the Unmet Needs model when exploring the relationship between depression, rejection of care, and behaviors directed towards others. The study found that changes in behaviors directed towards others were related to a lack of understanding, depression, and rejection of care. The authors postulated that the lack of understanding was caused by the progression of the dementia illness and resulted in increased behaviors of rejection of care and behaviors towards others [42]. In this case, the lack of understanding is the unmet need and is being expressed through the behavior of rejection of care. Other researchers have assessed this model within interventions designed to increase positive engagement [43], increase appropriate sensory techniques [45], improve standardized stimulation levels [33] and decrease pain and discomfort [9] for the ultimate goal of addressing a possible unmet need and thereby reducing disruptive behaviors.

The final theoretical model that will be discussed within this review includes The Stress Process Model (SPM) [3] and the Stress Process Model for Individuals with Dementia (SPM for IWDs) [46]. The SPM was originally developed to examine the stress process of caregiving for PWDs and is one of the primary paradigms for understanding the relationship between stress and health. The SPM for IWDs is a more recent model based on the SPM for caregivers and was developed specifically to examine the illness experience and associated stress process of living with dementia. The advantage of these models is the incorporation and integration of both the micro and macro level relationships between background characteristics, stressors, resources, and outcomes of well-being [47]. The models incorporate the following domains; (1) background and care context (i.e. age, gender, education, occupation), (2) objective (i.e. cognitive status, functional dependency, behaviors) and subjective (i.e. distress) primary stressors, (3) secondary roles (i.e. family conflict, work conflict) and intra-psychic strains (i.e. self-esteem, mastery), and (4) outcomes of well-being (i.e. depression, anxiety, quality of life) $[3,46]$. The model also incorporates the role of mediators as a domain that include coping and social support resources which are important in examining differences among caregivers and/or PWDs $[3,46]$. As a whole, this model illustrates how primary objective and subjective stressors impact secondary roles and intra-psychic strain outcomes and collectively how these domains effect well-being outcomes along with how this process is mediated by other factors.

Multiple studies have examined the impact of caregiving and stress on various aspects of health and well-being using the SPM and the SPM for IWDs. With the specific focus on the primary objective stressor of behaviors, researchers testing this model can assess the role by which the objective primary stressor of behaviors influences subjective stressors, secondary strains, and outcomes of well-being of caregivers $[48,49]$. Understanding this process allows for the development of nonpharmacological interventions related to behaviors and caregivers distress. This can be seen with a 
study conducted by Farran and colleagues [50] who tested a caregiver skill building intervention on reducing caregiver emotional distress and agitated behaviors of PWDs. Results found that psychoeducation, support, and skill building interventions were effective in decreasing emotional distress in dealing with behaviors. Additionally, Judge, Yarry, Looman and Bass [51] examined the efficacy of a dyadic intervention using a Strength-Based Approach combining educational and cognitive rehabilitation skills training in order to address dyadic care needs and issues. This intervention, guided by the SPM and the SPM for IWDs, significantly improved caregiver's emotional heath strain, dyadic relationship strain, role captivity (i.e. feelings of being trapped in the caregiving role), and mastery as well as significant reductions in symptoms of depression and anxiety for caregivers [51].

Although the SPM for IWDs has not received extensive investigation, several studies that have used the model have found interesting and insightful results. These results serve as a useful heuristic for understanding the illness experience and provide evidence for the inclusion of PWDs in the research process along with guidelines for implementing self-report methodology with PWDs [52]. Specifically, in one study, the SPM for IWDs was used to test for significant relationships among the model domains. Results found that PWDs who self-reported: 1) more embarrassment about their memory problems experienced greater symptoms of anxiety; 2 ) increased feelings of role captivity (i.e., feelings of being trapped in the care receiver role) and physical health strain experienced more depressive symptoms; and 3) fewer difficulties with completing instrumental activities of daily living and greater feelings of self-efficacy experienced better quality of life [53]. In another study, the SPM for IWDs was used to organize responses from open-ended interview questions conducted with PWDs that focused on their perceptions of living with the illness. Key findings centered on the impact of living with dementia that included both negative and positive feelings such as feelings of embarrassment, loss of one's sense of self, difficulties managing cognitive and functional abilities, concerns about the progression of the illness, and improved interpersonal relationships [54]. Finally, the model was used to examine quality of life in PWDs. Results found that everyday decision making and negative dyadic strain were unique predictors of quality of life [55]. Such that, PWDs who selfreported they were more involved in providing input about daily decisions reported better quality of life and PWDs who self-reported they had fewer negative interactions with their caregivers had better quality of life as well. Results such as these, which are grounded within the SPM for IWDs, are evidence supporting the fact that many of the cognitive, psychological, emotional, behavioral, and functional symptoms exhibited by PWDs cannot simply be discounted due to the individual having a dementing illness. Rather, these symptoms are a reflection of the subjective experience in which PWDs are encountering. Therefore, it can be postulated that the illness experience could help explain the needs that PWDs are experiencing as well as those needs that are currently being unmet which could be the underlying influence driving PWDs to exhibit various behaviors.

Across each of the conceptual models reviewed, there is a common theme relating to the identification of needs of PWDs. In some models this connection to needs is very evident as in the Unmet Needs model and the NDB model. This theme also can be identified within the SPM/SPM for IWDs as needs may be a mediating factor between objective primary stressors (i.e. behaviors) and various outcomes $[46,56]$. For the PLST model, identifying the triggers or stressors (or unmet needs) within the environment such as stimulation, activities, or physical discomfort may help alleviate behaviors. Understanding the possible needs of PWDs has been deemed vital as they formulate the basis for developing non-pharmacological interventions [20,31]. Even more, the shift to a PCC 
approach for addressing behavioral issues allows for the possibility to proactively address behaviors before becoming an 'unmet' need and being ascribed as solely an issue due to the underlying dementia pathology [8]. Given the importance of understanding behaviors of PWDs, the next two sections will review current methods and techniques used to measure behaviors experienced by PWDs and non-pharmacological approaches for intervening upon select behaviors that emphasize the identification of needs of PWDs, respectively.

\section{Measurement Techniques}

In order to understand and quantify the range of behaviors experienced by PWDs, reliable and valid measures are necessary. Proper measures of behaviors are crucial for understanding the impact of behaviors across the illness, analyzing effects of non-pharmacological interventions, and continued monitoring of the relationships between behaviors and various outcome measures [57]. The behavioral assessment scales that have been utilized within previous literature vary widely in measurement focus (i.e. clinical or research based), scope of behaviors (i.e. a narrow focus on one specific behavior or a comprehensive span of general problem behaviors), and rating method (i.e. direct patient observation by clinician and/or trained researcher or family caregiver's proxy-reports) [57].

Within the dementia literature, several behavioral rating scales have been developed that focus on the behavior of agitation. The behavior of agitation has received extensive investigation as it is a distressful and impactful behavior experienced by PWDs. Two commonly used rating scales include The Cohen-Mansfield Agitation Inventory (CMAI) [58] and the Agitation Behavior Mapping Instrument (ABMI) [58]. Both measures focus on the frequency of agitated behaviors and use caregiver proxy-reports [59]. The CMAI uses a 7-point scale that rates the frequency of different types of agitated behaviors. The CMAI was originally developed for research purposes within a nursing home setting, however, this scale also has been used in clinical settings as well [59]. The CMAl, shown to be both highly reliable and valid $[58,60]$, has provided researchers the ability to assess the varying events preceding expressions of agitated behaviors as well as environmental factors associated with agitation. For instance, Bédard and colleagues [31] utilized the CMAl to assess PWDs who were participants in a need-based intervention to reduce verbal agitation. Similarly, this measure has been employed in numerous intervention studies assessing a wide range of protocols including the use of a personalized simulated presence [10], personalized meaningful activities [43], and animal-assisted interventions [61, 62] in order to study the effects of interventions on reducing agitated behaviors in PWDs.

Additional specific measurement scales within the literature include the Apathy Evaluation Scale (AES) [63] and the Resistiveness to Care Scale [64]. The AES characterizes and quantifies apathy in individuals who are 55 and older and has demonstrated good inter-rater and test-retest reliability specifically within the population of PWDs [64]. This measure is unique in its ability to offer both an AES-self-report, AES-informant rating, and AES-clinician rating, however, research assessing these various formats has found that the AES-self-report and AES-clinician rating offer little diagnostically above and beyond AES-informant rating [64]. The Resistiveness to Care Scale [63], which is an observation-based measure, was developed to examine the extent to which PWDs resisted or refused care, which is a common behavior experienced by PWDs residing within an institutionalized setting $[65,66]$. Resistiveness to care is distinctly different from agitation and aggression as it 
includes behaviors that interfere with the provision of necessary care for PWDs. In this case, resistiveness to care includes PWDs withstanding or opposing efforts of the caregiver and is thought to be due to PWD's lack of understanding of their caregiver's intentions [67].

Some researchers utilize behavioral measurements that employ a narrow focus on one specific behavior as the goal is to collect detailed and clinically relevant information for one specific behavior (i.e. CMAI and Resistiveness to Care Scale) [68]. Typically, the behavior of resistiveness to care has been linked in previous research to similar behaviors such as agitation or aggression [67]. However, the investigators who developed the Resistiveness to Care Scale have differing definitions of agitation compared to the investigators of the CMAI $[59,63]$. This distinction in definition and conceptualization of agitation is important to note in order for future researchers to choose the appropriate behavioral measure to frame and study their respective research question. However, this very distinction also highlights a limitation within the current literature which includes a lack of a unified conceptualization or 'gold standard' for specific behaviors [57].

As previously mentioned, there also are a subset of behavioral measures that take a more comprehensive approach in measuring behaviors of PWDs. The Neuropsychiatric Inventory (NPI) [69], one of the most widely known comprehensive behavior measures, assesses a range of behaviors experienced by PWDs (i.e., apathy, delusions, agitation, anxiety). The NPI provides a means for distinguishing frequency and severity of behaviors as rated by caregivers along with the associated distress experienced by caregivers for each behavior [69]. The NPI also can be used to assist in distinguishing the type or form of dementia an individual may have, such as Alzheimer's disease, dementia of Lewy-body, or frontotemporal dementia based on the frequency and severity of behaviors [69]. This is done through the expansive range of behaviors assessed including delusions, hallucinations, anxiety, apathy, agitation/aggression, disinhibition, euphoria, irritability/ lability, and aberrant motor activity [69]. The NPI has been used to study the effectiveness of a wide range of non-pharmacological interventions on reducing neuropsychiatric symptoms or BPSD. Some of these interventions include, conditional effects of caregiver social support and mastery of behaviors [49], the effects of individualized tailored activity programs on reducing neuropsychiatric symptoms [41] and the efficacy of music therapy on behaviors [70].

Another comprehensive behavioral measure includes the Behavioral Pathology in Alzheimer's Disease Rating Scale (BEHAVE-AD) [71], which is a behavioral identification and characterization measure that assess the nature of the behavioral disturbance pathology in Alzheimer's disease and related dementia [72]. Similar to the NPI, The BEHAVE-AD is mainly utilized within the clinical setting (i.e. pharmacological clinical trials) requiring a clinician to administer the scale and includes a range of behaviors such as anxieties and phobias, affective disturbances, nonspecific agitation, aggression, hallucinations and delusions, and diurnal rhythm disturbances [71]. Another comprehensive measure is The Revised Memory and Behavior Problems Checklist (RMBPC) [68] which assesses memory related, emotional related, and disruptive behaviors. The RMBPC was developed for clinical as well as research settings focusing on observable behaviors that could potentially be modified [68]. This measure includes the caregiver's report of frequency of behaviors and caregiver's reactions to behaviors (i.e. distress). Similar to the NPI, this allows researchers and clinicians to design individualized interventions addressing those person-specific behaviors that are most distressing to the caregiver [68]. This approach was used in research by Norton, Allen, Snow, Hardin, and Burgio [36], who found that certified nursing assistances who cared for PWDs who had more pain had higher levels of burden related to emotional behavior problems. Additionally, Hepburn, Lewis, 
Sherman, and Tornatore $[73,74]$ employed the RMBPC within a psychoeducation intervention to assess caregivers' reactions and well-being in relation to PWD's problem behaviors.

As evident, the literature provides a vast array of existing behavioral measures with varying behavioral foci, methods, and conceptualizations of behaviors. However, within this branch of literature, there are several difficulties within these measurement techniques [57]. One such issue includes operationalizing BPSD or behaviors exhibited by PWDs. Given that behaviors are not consistently experienced by all PWDs and that these behaviors can be influenced by individual differences in history, personality, and environments it is difficult for researchers and clinicians to provide universal definitions for various behaviors [57]. Even more, another methodological issue includes rater-bias [74]. A rater's knowledge of a PWD's cognitive impairment could influence the rater's behavioral assessment. Additionally, the distinction of what constitutes a 'problem' behavior is based on the rater's interpretation and experience with the behavior, which can vary widely depending on whether the rater is a personal caregiver and therefore interpretations are based on several weeks of observation, compared to researchers or clinicians' observations which may be based on only minutes to hours [57]. Additionally, caregiver's perceptions and experiences with behaviors may vary greatly from how a PWD may perceive and experience their own behavior. For example, a caregiver may perceive and experience repetitive question asking as very distressing due to the frequency of the behavior. Whereas the PWD may not perceive and experience this behavior as problematic nor as distressing. Conversely, a caregiver may not perceive and experience the behavior of apathy as distressing whereas the PWD may perceive and experience apathy as very problematic and distressing. Another limitation related to rater-bias stems from the relational aspect between family caregivers and PWDs. While many researchers may choose to employ a caregiver based informant rating due to the relative ease of this approach and comfort to the PWD compared to a third-party observer, family caregiver ratings may be influenced by other factors including the pre-existing nature of the relationship with the PWD [73], the caregiver's emotional health [75-77] and the caregiver's level of burden [74, 78]. These limitations stemming from proxyreports and/or observational data collection methods, may result in under- or over-estimations of the frequency and severity of the behavior(s) and also the level of distress experienced by the PWD.

Reviewing the behavioral measures discussed above, one commonality is the lack of direct participation and inclusion of PWDs. This is a very challenging issue that all researchers and clinicians in the field of dementia encounter and is primarily due to the role and impact of cognitive impairment on an individual's ability to provide self-report information. To date, across all behavioral assessments, behavioral frequency and severity and related perceived distress from behaviors are observed and reported by either a caregiver proxy-report, research observation, or clinician's assessment. This line of research has amassed findings that provide important information concerning a range of behaviors, the associated negative outcomes, and the relative distress caregivers experience. However, what researchers are not currently aware of is the level of distress PWDs feel in relation to the behaviors they exhibit. For example, do PWDs perceive behaviors the same as their caregivers? Do these behaviors impact PWD's own self-rated quality of life or depressive symptoms? Are there certain behaviors that do not cause distress to PWDs despite causing distress for caregivers? How do distressing behaviors vary across PWDs? Could interventions be developed in order to address not only the caregiver's distress from BPSD but also PWD's distress from BPSD? These are all important questions that have the potential to extend the literature on dementia and BPSD. However, this review did not find any published studies that used self-report 
measures of behaviors directly with PWDs. Given the prior discussion regarding findings that support the inclusion of PWDs in the research process and the value in implementing self-report data collection protocols with PWDs [52-55], it is imperative that this is extended to the measurement of behaviors. As such, future research should examine the experience and impact of behaviors from the PWD's perspective using self-report data collection methods. The next section will discuss key findings from non-pharmacological interventions along with future directions that focus on actively including PWDs as part of the research and intervention process.

\section{Non-Pharmacological Interventions}

As previously mentioned, behavioral symptoms in dementia are consistently reported as one of the most stressful aspects of care [44]. There is, however, a plethora of literature that has developed and examined non-pharmacological interventions available for PWDs and their caregivers. These interventions vary in the approach utilized with some protocols directly targeting or addressing the behavior(s) exhibited by the PWD with the goal of reducing or eliminating the behavior(s). While other protocols indirectly target or address the behavior(s) through the caregiver with the goal of reducing caregivers' distress to the behavior(s) and thereby indirectly positively impacting the behaviors experienced by PWDs. The next section will provide a description and brief research overview of the following types of non-pharmacological interventions that have been categorized as the following: behavioral interventions, alternative care interventions, engagement-based interventions, and caregiver interventions. Although there is some overlap between our intervention categories, our goal was to highlight and categorize different types of nonpharmacological protocols according to the: type of behavior(s) addressed; illness stage/symptom severity (i.e., mild, moderate, severe); implementation setting (i.e., institutionalized, communitydwelling; target of the intervention (i.e., PWD, caregiver, or both); design of the intervention (i.e., single or multi-component); type of outcome(s) measured; and amount of research evidence demonstrating efficacy of the intervention.

\subsection{Behavioral Interventions}

Within behavioral interventions that target PWDs, behaviors are the critical outcome measure being tested. This is primarily accomplished by identifying the need or stimuli that is linked to the behavior(s) [1]. For instance, some behavioral interventions identify the need for active engagement [79], sensory stimulation [80], or social stimulation [33] to effectively eliminate or reduce a particular behavioral symptom. Focusing on addressing the behavior(s) that the PWD is exhibiting through intervention, in theory, should improve behavioral symptoms once the need is met or stimulus is addressed. Addressing the underlying component contributing to the behavior(s) in this manner leads many behavioral interventions to be single component designs, as compared to a multi-component design, that focus on one element (i.e. increase social stimulation to decrease agitation) that is being intervened upon to reduce a specific behavior $[31,33,80]$.

These types of behavioral interventions tend to focus on developing interventions for PWDs [24, $33,42]$ who are in the moderate to severe stages of the illness [36, 81] and primarily living in nursing homes or long-term care facilities [33, 82, 83]. Typically, behavioral interventions target a specific behavior such as agitation $[31,82]$ or rejection of care $[42,49]$. Few behavioral studies address the changes in behaviors across the different stages of the illness, those that do use observational 
measurement scales such as the NPI and RMBPC (which are administer by researchers or trained staff).

As previously mentioned, agitation is one of the most researched behaviors of PWDs and countless studies have attempted to develop behavioral interventions to reduce or eliminate agitated behaviors. Cohen-Mansfield and colleagues [33] developed an intervention using various forms of stimuli (music, social stimuli, individualized stimuli) in order to reduce agitation. Through the use of a Self-Identity Questionnaire, the researchers attempted to determine those activities that were enjoyable to the PWD before diagnosis as well as those activities that they currently enjoyed. After direct observation via the ABMI, the frequency of agitated behaviors was coded by trained research assistants. The study concluded that music, social, and individual stimuli based on the PWD's self-identity were associated with decreased agitation [33].

Other behavioral interventions targeting agitation have found improvements in agitated behaviors through the use of meaningful activities. One such example is the Continuous Activity Program, which incorporates continuous structured and meaningful activity between PWDs and a staff member [24]. The Continuous Activity Program was found to have a wide range of positive outcomes such as decreased use of psychotropic medication, improvements in nutritional states, increased family satisfaction, and decreased agitation and improved sleep among PWDs [24]. Similarly, an intervention known as SimPres (Simulated Presence) uses the personalized approach of having an individual record a detail-rich memory for a PWD in order to effectively simulate a caller's presence on the telephone to improve psycho-emotional well-being and resolve agitated and withdrawn behaviors [10]. This program found extremely positive results as compared to placebo groups with intervention groups showing improvements in both agitated and withdrawn behaviors [10]. All of these behavioral interventions were designed to address the single behavior of agitation by addressing the need for meaningful activity or stimulation. However, these interventions did not examine potential differences in behaviors across the illness, as the studies included PWDs with more severe symptoms of dementia, and did not include community-dwelling PWDs.

\subsection{Alternative Care Interventions}

Another type of non-pharmacological intervention that has been employed to address behaviors will be referred to as 'alternative' care interventions. Alternative care interventions, like behavioral interventions, directly target the PWD in order to reduce or eliminate behaviors. However, alternative care interventions focus on those alternative forms of care, including art, music, animalassistance, massage and more that facilitate health and stimulates positive feelings and well-being within the PWD [61]. Alternative care interventions take an individualized approach, more so than other forms of interventions, by personalizing aspects of stimulation, meaningful activities, and engagements for PWDs [43]. Interventions within this category vary from addressing behaviors of agitation through studies utilizing animal assisted therapy, increased social contact, and even music therapy [33] to other sets of behaviors, such as rejection of care [42], withdrawn behaviors [10], physically aggressive behaviors [82], and even depressed behaviors [67]. In comparison to behavioral interventions, alternative care interventions address a potential underlying need(s) of PWDs and develop interventions that facilitate engagement in one's care. This is primarily accomplished with sensory stimulation and/or meaningful activities. For example, interventions 
using multi-sensory stimulation or motor stimulation have found increased participation in activities, improvements in BPSD, and improved communication [61, 70, 83]. Similarly, interventions focused on increasing meaningful or purposeful activity has observed reduced disruptive behaviors, increased social connections with other residents, and increased positive affect and engagement $[45,84-86]$.

Similar to behavioral interventions, alternative care interventions are typically single component interventions $[45,86]$, targeting PWDs in the moderate to severe stages of the illness process [66, 86] living in nursing home or long-term care facilities [10, 87]. However, differing from behavioral interventions, the research to date on alternative care interventions have typically lacked aspects of rigorous methodological designs including low sample sizes and lack of an appropriate control group for comparison. Furthermore, research has not found evidence to suggest these types of interventions are efficacious, which may stem from the methodological issues mentioned. For instance, one study assessed the use of robotic cats as a form of animal-assisted intervention to provide social support and reduce BPSD in PWDs in the severe stages of the illness. Case studies of four PWDs were analyzed and findings reported from professional caregivers and relatives indicated the robotic cat opened a line of communication when caring for the PWD as well as being a sedative, soothing, and comforting influence for the PWD [61]. The researchers also claimed that participants had less agitated behaviors and better quality of life. However, though agitation was measured using a reliable and valid scale (CMAI) it cannot be concluded that this approach was efficacious due to the small sample size and lack of an appropriate comparison group. Although these results provide a starting point, more methodologically rigorous research is needed in order to conclude that these types of intervention programs positively impact behaviors experienced by PWDs and their caregivers. These methodological issues can be seen within other studies which conclude findings based on results that are from caregivers or staff stating their personal experiences in noticing differences within individuals rather than results that are based on statistically significant findings [84, 87]. There are, however, a few noted exceptions of alternative care interventions that have employed a randomized control trial design and have found sufficient efficacy. One such intervention was conducted by Raglio and colleagues [70] that assessed the efficacy of music therapy in the treatment of BPSD. Participants were randomly assigned to the experimental group where they received three cycles of ten music therapy sessions (30 min/session) or the control group that underwent educational and entertainment activities. Over the course of eight and then sixteen weeks, there was a significant decrease in global NPI scores for the experimental group but not the control group. This effect for the experimental group also persisted over the course of four weeks after the end of treatment and was more relevant for improving agitation, delusions, anxiety, apathy, irritability, aberrant motor activity and nighttime disturbances [70].

\subsection{Engagement-Based Interventions}

Similar to both behavioral and alternative care interventions, interventions designed to target engagement have found beneficial results concerning behaviors by providing structured opportunities to participate in purposeful and meaningful activities. One such example is Montessori-based activities [43, 88], that is based on the work of Maria Montessori who taught cognitive, social, and functional skills to children. These same principles have been found to be beneficial for PWDs to participate in activities regardless of their level of cognitive impairment [88]. 
Montessori-based activities are tailored to the level of each PWD in order to promote full participation from individuals by: capitalizing on PWD's remaining cognitive abilities; compensating for PWD's cognitive difficulties by using environmental cueing and aids; and providing appropriate, structured and purposeful activity programming with the result of indirectly reducing behavioral disturbances in PWD $[79,88]$. As with behavioral and alternative care interventions, Montessoribased interventions directly target the PWD. These studies typically utilize a within-subjects design or a randomized controlled trial design and collect multiple observational data points across time; which addresses the common issues related to the use of an appropriate comparison group and small sample size, respectively.

To illustrate, Judge, Camp, and Orsulic-Jeras [79] used a randomized controlled trial design to examine the efficacy of Montessori-based individual and group activities on different types of engagement within an adult day care setting. Constructive engagement, passive engagement, nonengagement, and self-engagement were all evaluated in both the experimental group (Montessoribased activities) and control group (engaged in regularly scheduled program activities). Constructive engagement was conceptualized as the more positive and beneficial form of engagement as compared to passive, self, and/or non-engagement. Results found PWDs showed significantly more constructive engagement when participating in Montessori-based activities as compared to regularly scheduled programs. Additionally, passive engagement was reduced compared to regularly scheduled programs in some situations. One of the underlying principles of this type of intervention approach targeting engagement is the idea that if PWDs are constructively engaged then they will not be engaged in disruptive or distressful behaviors, that also include negative forms of engagement such as self- and non-engagement. The study assessed participant agitation using the CMAI, however, results for agitation were not reported. Further research is needed to examine whether engagement-based interventions also reduce certain disruptive and/or distressful behaviors experienced by PWDs.

To address this issue, Van der Ploeg and colleagues [43] tested a personalized one-on-one intervention using interactive activities based on Montessori principles to improve agitation, affect, and engagement. Within this study, levels of observed agitation, affect, and engagement were assessed before, during, and after personalized activities were delivered. The intervention condition was compared to a control group who experienced engagement through basic conversation and newspaper stories. Results showed that both the Montessori group and control group experienced decreased agitation during times of activity compared to baseline. However, this finding was stronger for participants in the Montessori group as compared to participants in the control group. Collectively these findings $[43,79]$ demonstrate the beneficial results of implementing intervention programs that facilitate positive or beneficial engagement for PWDs and highlight the possibility of also reducing agitation. Additional research is needed to examine whether engagement-based interventions positively impact other types of behaviors experienced by PWDs (i.e., anxiety, apathy) and potentially improve psychosocial well-being outcomes for PWDs, such as quality of life.

\subsection{Caregiver Interventions}

As behavioral, alternative care, and engagement-based interventions have provided many important findings to the existing literature on how to intervene upon the behaviors of PWDs, researchers also have looked at behaviors from the perspective of caregivers. Whereas 
interventions targeting PWDs aim to directly eliminate or reduce the behavior(s), interventions targeting caregivers aim to provide caregivers with the education and tools to manage problem behaviors and/or change their perspective of behaviors. In order to accomplish this aim, educational and skill training interventions have been developed to provide caregivers with education concerning the disease process and progression, psychosocial and emotional support, and skills training to manage and cope with behaviors. A majority of these interventions focus on educational aspects including changes in cognitive functioning and communication abilities as well as management of behavioral issues [44]. This style of intervention not only educates caregivers on the impending changes associated with a dementia diagnosis but also provides training on how communication styles, appraisals of situations, and daily activities must be adapted and new methods utilized for more effective interactions with the PWD [44]. Specifically, educational and skills training interventions that are developed for managing behavioral problems teach caregivers the skills that are thought to effectively address and manage the behaviors of PWDs [89]. Additionally, other interventions aim to teach caregivers to modify their own psychosocial and emotional responses to these behavioral challenges by reducing their anger, frustration, and stress and increasing their successful problem solving and engagement in pleasant activities [89].

Compared to the interventions discussed previously, the majority of caregiver interventions are multi-component interventions, meaning the protocols include two or more elements and typically target a wider range of study outcomes. For example, many caregiver interventions for addressing behavioral issues include educational information along with communication and skill building techniques for coping and managing the myriad of symptoms and care-related issues associated with dementia. As such, most of the caregiver interventions are designed to target and improve a wide range of outcomes experienced by caregivers that include behaviors but also other key outcomes such as caregiver burden, caregiver symptoms of depression, and caregiver quality of life. With respect to the method of delivery, most of the caregiver interventions do not actively include the PWD but rather the focus is targeted at caregivers $[49,89,90]$. To date, only a few studies have included both the PWD and the caregiver as active participants in the intervention program [51, 91], where both individuals participate together in the program and study outcomes are collected directly from both PWDs and caregivers.

With respect to the type of research design used, many caregiver interventions implement a randomized controlled trial (RCT) to evaluate efficacy, which is considered the gold standard research design for rigorous scientific evaluation [92]. These RCTs generally examine the effects that problem behaviors have on caregiver outcomes (i.e. caregiver burden, stress, quality of life) and how the multi-component intervention was able to eliminate or decrease the frequency of problem behaviors and therefore improve caregiver outcomes through the examination of pre and post-tests [89]. Additionally, these interventions have been able to evaluate outcomes across the dementia illness experience from mild to moderate to severe stages and include PWDs that live within the community [51, 90, 93, 94] and to a lesser extent, PWDs in nursing homes or long-term care facilities [95]. Lastly, these types of caregiver interventions have been found to be highly successful and efficacious in improving caregiver related outcomes and in providing caregivers with core skills for managing and coping with behaviors $[40,41,48,51,73,90,92-95]$.

One such caregiver intervention is the Tailored Activity Program (TAP) [40]. Using a RCT design, this activity based program was designed to identify and reduce behavioral disturbances in PWDs. With multiple outcome measures, this study assessed behavioral occurrences in PWDs and 
engagement as well as caregiver burden, mastery, self-efficacy, and the use of simplification strategies to manage behaviors [40]. The results showed a treatment effect for frequency of behavioral occurrences reported by caregivers and a reduction in the number of caregiver reports of agitation and argumentative behaviors by caregivers who received TAP as compared to caregivers in the control condition. Additionally, caregivers reported greater levels of mastery, enhanced selfefficacy using activities, and greater use of simplification techniques compared to controls [40]. The TAP caregiver intervention illustrates the effectiveness of education and skills training at reducing behavioral problems while also improving caregiver outcomes.

Another successful caregiver intervention is the Savvy Caregiver Program, which was developed and field tested by Hepburn, Lewis, Sherman and Tornatore [73]. The Savvy Caregiver Program is a psychoeducational program that prepares and trains caregivers for the unfamiliar role of caregiving. The program uses trained facilitators to work with family caregivers of PWDs by providing them with knowledge, skills, and attitudes that are needed to carry out such a role. Caregivers that have completed the program have shown improvement in their: 1) reactions to behaviors exhibited; 2 ) caregiver burden; and 3) beliefs about caregiving [73]. This study highlights an interesting finding that is common for many of the caregiver interventions (for exceptions see $[41,48]$ ), in that the specific behavior(s) of PWDs are not reduced or eliminated but rather caregiver's ability to manage, redirect, and/or reappraise their thinking about the behavior(s) has changed [50, 73, 93-97]. This is very important, as it illustrates the benefits of indirectly intervening and the positive impact experienced by caregivers (i.e., changing the way one manages or thinks about a behavior results in beneficial outcomes). However, the potential negative impact for PWDs is not readily addressed nor impacted. Furthermore, there is not sufficient evidence yet to understand how PWDs perceive, appraise, and experience their behaviors. This is an important area within the research literature that warrants further investigation. Specifically, understanding the vast array of behaviors as subjectively experienced by PWDs and the resulting distress may provide significant and important information about the illness experience and, therefore, how to best intervene. Currently, the literature does not provide clear methodological or conceptual guidelines for how to best conduct this type of research. For example, when can PWDs provide reliable and valid self-report data about their illness experience? And what types of structured questions and response options are best suited for conducting self-report data collection with PWDs? Researchers have only begun to address these important issues [52].

\section{Future Directions}

There is a tremendous amount of existing literature on the types of behaviors PWDs experience, the possible effects these behaviors have on caregivers, as well as interventions that aid formal and informal caregivers in the task of managing these behaviors and interventions that directly reduce or eliminate behaviors exhibited by PWDs. The purpose of this article was to provide an overview of the significance of behaviors experienced by PWDs along with a discussion of several conceptual models and measurement techniques used to study behaviors experienced by PWDs. And to categorize and discuss different types of non-pharmacological interventions used to address the wide range of behaviors. Lastly, the goal of this paper was to reflect on potential gaps within the research literature and provide thoughts about future directions. 
One gap identified within the literature is the absence of self-report data from PWDs that provides information about their subjective experience in coping and managing with the behaviors they experience. For example, the theoretical models reviewed each include PCC principles in how behaviors are conceptualized, such as the role and importance of an individual's personal preferences, their background and care context, environmental stressors, and/or available resources. Compared to a traditional medical model, PCC is a holistic approach that provides a means for understanding the 'whole-person' [98]. Behaviors of PWDs illustrates this distinction very well. In the traditional perspective, behaviors are used to describe actions exhibited by PWDs and implies an undesirable symptom or pathology of the illness that requires intervention [98]. On the other hand, a PCC approach would view these actions as personal expressions or a means by which to communicate and/or convey a need [98]. Given the progression of dementia results in changes in cognitive and functional abilities, many of the internal states, desires and feelings expressed by an individual may be conceptualized by others as solely "undesirable behaviors" that need to be eliminated or reduced which only serves to medicalize the experience of living with a chronic illness [98]. For example, much of the prior literature has framed behaviors with this lens of "difficult" or "problematic" and yet these descriptions of behaviors are based on the perceptions and appraisals of caregivers and not from self-report data collected from PWDs. The lack of PCC approach also is evident in the measurement techniques used, as the measures used to date do not incorporate or measure the PWD's subjective experience in how they perceive, appraise, cope with and manage their behaviors. Clearly this challenge stems from difficulties in conducting research with individuals who have a cognitive impairment such as dementia. The lack of a true PCC approach within research on PWDs also has been identified as an important issue within the healthcare system according to the 2001 Institute of Medicine Summit [98]. Additionally, in the recent 2016 Research Summit on Dementia Care two recommendations for future research included: 1) involving PWDs and caregivers as members of the research team and 2) involving PWDs as study participants [99].

In order to address this gap, researchers have begun to include PWDs in the research process and as study participants by collecting self-report data from PWDs [51-55, 91]. For example, several of these studies have focused on interviewing individuals in the mild to moderate stages of the illness about their subjective experiences in living with dementia [53-55]. Several important findings emerge that expand our knowledge base about living with dementia and challenge previously held beliefs and bias. First, these studies have found that individuals with mild to moderate symptoms of dementia were able to self-report about their own subjective illness experience and provide reliable and valid data. This is a very important finding as it challenges prior assumptions about PWD's ability to provide insightful and meaningful information about their experience in living with dementia. Secondly, research has found that PWD's perceptions and experiences can differ from the proxy-report data collected from their family caregivers [73, 74, 76-78]. Coupled with the prior finding, this highlights the importance of collecting data directly from PWDs whenever possible as caregivers may be under- or over-estimating their perceptions of their loved one's illness experience. Third, by collecting self-report data directly from PWDs, researchers and clinicians have a new lens for understanding the illness experience and, perhaps more importantly, for developing and implementing more targeted and impactful interventions for PWDs.

Given the steady rise in including PWDs in the research and intervention process, a next step would be to extend this to the literature on behaviors experienced by PWDs. Specifically, no published studies to date have assessed the self-reported behaviors of PWDs in order to attempt to 
understand how these individuals perceive and acknowledge the behaviors they are experiencing, the related distress derived from those behaviors, and how PWDs manage and cope with their behaviors. Initiating this process could potentially provide researchers with a greater understanding of behavioral expressions, the underlying needs that are attempting to be communicated, and guidance on how to develop interventions to reduce distress from behavioral expressions. For example, understanding the level of distress PWDs are experiencing through self-report measures could have major implications for the design of behavioral interventions, including what behavioral expressions are targeted. Additionally, given that many of the non-pharmacological interventions and conceptual models previously discussed incorporate the idea of addressing an unmet need, including data collected directly from PWDs may be very useful in identifying unmet needs. For example, several studies have examined self-reported unmet needs and found that PWDs identify more unmet needs as compared to proxy-reports from their caregivers and there were inconsistencies in the types of unmet needs identified by self-report verses proxy-report [100, 101]. More research is needed to further understand the self-reported unmet needs of PWDs and how this can inform the development of non-pharmacological interventions that address PWD's unmet needs, behavioral expressions, and any resulting distress.

Another gap within the current behavioral literature includes the lack of research addressing all stages of the dementia illness, including mild, moderate, and severe stages. A majority of the research on behaviors is limited to the later stages of the dementia illness when cognitive, functional, and behavioral impairments are the greatest. Restricting research to the subsets of behaviors that are most frequent during moderate to severe stages and behaviors that are reported as most distressing to caregivers does not provide a comprehensive overview of the range of possible behavior experienced by PWDs. Future research needs to assess behavioral expression that are distressing to both caregivers and PWDs across the continuum of the illness. For example, most studies on unmet needs focus on those aspects of unmet needs that caregivers are identifying, such as mobility issues and incontinence $[100,101]$, which typically are issues in the moderate to severe illness stages. Research has shown that when PWDs self-report and identify their own unmet needs across illness stages, they identify differing needs including psychological distress, environmental discomfort, and preferences with care $[100,101]$. This suggests that in order to fully understand the illness experience future research needs to incorporate a variety of needs that may be expressed by PWDs, not solely caregivers, and that vary by stage of illness. Acknowledging that behavioral expressions and unmet needs can occur at any stage of the illness process, can vary in complexity, and can vary from person to person is critical when considering behavioral research that incorporates the self-reported experience of PWDs.

In considering this information, one potential direction for further study that would address the aforementioned gaps within the literature on behaviors, would be to conduct studies that increase researchers understanding about the illness experience and provide empirical evidence for the selfreported behavioral expressions of PWDs. Such studies would, when possible, include the use of self-report measures of PWDs ranging from mild, moderate, and severe stages, and include theoretical frameworks that are based on the illness experience, such as the SPM for IWDs [46]. For instance, using the SPM for IWDs, the objective primary stressors (i.e., behavior frequency) and subjective primary stressor (i.e., behavioral distress) could be examined to determine the direct impact on the outcome of well-being, such as quality of life as well as the indirect effect on depression when mediated by differing unmet needs identified by the PWD. Understanding the 
potential predictive power of unmet needs on both behavioral expressions and outcomes of wellbeing could aid in the development of interventions that specifically target unmet needs and also would reduce distress related to behavioral expressions. There is still much to learn from PWDs concerning the multi-dimensional experience of living with dementia and not all of these unanswered questions will or can be addressed seamlessly. However, it is essential that researchers take these next steps in moving the field forward by attempting to provide the means and methodology to examine the experiences that may be directly impacting the quality of life for PWDs and to truly encapsulate behavioral research using a PCC approach.

\section{Author Contributions}

MM conceived of the premise for the review paper, worked with $\mathrm{KJ}$ in conceptualizing the sections of the review paper, conducted the literature review for the paper, wrote the manuscript, and integrated feedback and input from KJ for each draft of the manuscript.

$\mathrm{KJ}$ worked with $\mathrm{MM}$ in conceptualizing the sections of the review paper, provided feedback and input for each draft of the manuscript, and reviewed and edited the final version of the manuscript.

\section{Competing Interests}

The authors have declared that no competing interests exist.

\section{References}

1. Desai AK, Grossberg GT. Recognition and management of behavioral disturbances in dementia. Prim Care Companion J Clin Psychiatry. 2001; 3: 93-109.

2. McKeith I, Cummings J. Behavioural changes and psychological symptoms in dementia disorders. Lancet Neurol. 2005; 4: 735-742.

3. Pearlin LI, Mullan JT, Semple SJ, Skaff MM. Caregiving and the stress process: An overview of concepts and their measures. Gerontologist. 1990; 30: 583-594.

4. Burgio L. Direct observation of behavioral disturbances of dementia and their environmental context. Int Psychogeriatr. 1997; 8: 343-346.

5. Kales HC, Gitlin LN, Lyketsos CG. Assessment and management of behavioral and psychological symptoms of dementia. BMJ. 2015; 350: h369.

6. Finkel SI, Costo e Silva J, Cohen G, Miller S, Sartorius N. Behavioral and psychological signs and symptoms of dementia: A consensus statement on current knowledge and implications for research and treatment. Int Psychogeriatr. 1997; 8: 497-500.

7. Algase DL, Beck C, Kolanowski A, Whall A, Berent S, Richards K, et al. Need-driven dementiacompromised behavior: An alternative view of disruptive behavior. Am J Alzheimers Dis. 1996; 11: 10-19.

8. Cohen-Mansfield J. Nonpharmacologic interventions for inappropriate behaviors in dementia: A review, summary, and critique. Am J Geriatr Psychiat. 2001; 9: 361-381.

9. Cohen-Mansfield J, Dakheel-Ali M, Marx MS, Thein K, Regier NG. Which unmet needs contribute to behavior problems in persons with advanced dementia? Psychiatry Res. 2015; 228: 59-64. 
10. Camberg L, Woods P, Ooi WL, Hurley A, Volicer L, Ashley J, et al. Evaluation of simulated presence: A personalized approach to enhance well-being in persons with Alzheimer's disease. J Am Geriatr Soc. 1999; 47: 446-452.

11. Moak GS. Characteristics of demented and nondemented geriatric admissions to a state hospital. Psychiatr Serv. 1990; 41: 799-801.

12. Kales HC, Chen P, Blow FC, Welsh DE, Mellow AM. Rates of clinical depression diagnosis, functional impairment, and nursing home placement in coexisting dementia and depression. Am J Geriatr Psychiatry. 2005; 13: 441-449.

13. Yaffe K, Fox P, Newcomer R, Sands L, Lindquist K, Dane K, et al. Patient and caregiver characteristics and nursing home placement in patients with dementia. JAMA. 2002; 287: 20902097.

14. Finkel S. Introduction to behavioural and psychological symptoms of dementia (BPSD). Int J Geriatr Psychiatry. 2000; 15: S2-S4.

15. Allen-Burge $R$, Stevens AB, Burgio LD. Effective behavioral interventions for decreasing dementia-related challenging behavior in nursing homes. Int J Geriatr Psychiatry. 1999; 14: 213-228.

16. Everitt DE, Fields DR, Soumerai SS, Avorn J. Resident behavior and staff distress in the nursing home. J Am Geriatr Soc. 1991; 39: 792-798.

17. Block C, Boczkowski JA, Hansen N, Vanderbeck M. Nursing home consultation: Difficult residents and frustrated staff. Gerontologist. 1987; 27: 443-446.

18. Lyketsos CG, Steinberg M, Tschanz JT, Norton MC, Steffens DC, Breitner JC. Mental and behavioral disturbances in dementia: Findings from the Cache County Study on Memory in Aging. Am J Psychiatry. 2000; 157: 708-714.

19. Lyketsos CG. Neuropsychiatric symptoms (behavioral and psychological symptoms of dementia) and the development of dementia treatments. Int Psychogeriatr. 2007; 19: 409-420.

20. Cohen-Mansfield J, Dakheel-Ali M, Jensen B, Marx MS, Thein K. An analysis of the relationships among engagement, agitated behavior, and affect in nursing home residents with dementia. Int Psychogeriatr. 2012; 24: 742-752.

21. Cohen-Mansfield J, Marx MS, Rosenthal AS. A description of agitation in a nursing home. J Gerontol Med Sci. 1989; 44: M77-M84.

22. Cohen-Mansfield J, Werner P. Environmental influences on agitation: An integrative summary of an observational study. AJADD. 1995; 10: 32-39.

23. Camp CJ, Cohen-Mansfield J, Capezuti EA. Mental health services in nursing homes: Use of nonpharmacologic interventions among nursing home residents with dementia. Psychiatr Serv. 2002; 53: 1397-1404.

24. Volicer L, Simard J, Pupa JH, Medrek R, Riordan ME. Effects of continuous activity programming on behavioral symptoms of dementia. J Am Med Dir Assoc. 2006; 7: 426-431.

25. Galynker II, Roane DM, Miner CR, Feinberg TE, Watts P. Negative symptoms in patients with Alzheimer's disease. Am J Geriatr Psychiatry. 1995; 3: 52-59.

26. Reichman WE, Coyne AC, Amirneni S, Molino JB, Egan S. Negative symptoms in Alzheimer's disease. Am J Psychiatry. 1996; 153: 424-426.

27. Mace NL. Principles of activities for persons with dementia. Phys Occup Ther Geriatr. 1987; 5: 13-28. 
28. Colling KB. Caregiver interventions for passive behaviors in dementia: Links to the NDB Model. Aging Ment Health. 2004; 8: 117-125.

29. Kovach CR. Handbook of pain and palliative care: Biobehavioral approaches for the life course. New York: Springer Science + Business Media; 2012. pp. 131-144.

30. Volicer L, Van der Steen JT, Frijters DH. Modifiable factors related to abusive behaviors in nursing home residents with dementia. J Am Med Dir Assoc. 2009; 10: 617-622.

31. Bédard A, Landreville $P$, Voyer $P$, Verreault $R$, Vézina J. Reducing verbal agitation in people with dementia: Evaluation of an intervention based on the satisfaction of basic needs. Aging Ment Health. 2011; 15: 855-865.

32. Schneider LS, Dagerman KS, Insel P. Risk of death with atypical antipsychotic drug treatment for dementia: Meta-analysis of randomized placebo-controlled trials. JAMA. 2005; 294: 19341943.

33. Cohen-Mansfield J, Marx MS, Dakheel-Ali M, Regier NG, Thein K, Freedman L. Can agitated behavior of nursing home residents with dementia be prevented with the use of standardized stimuli? J Am Geriatr Soc. 2010; 58: 1459-1464.

34. Kitwood T. The experience of dementia. Aging Ment Health. 1997; 1: 13-22.

35. Camp CJ, Bourgeois MS, Erkes J. Person-centered care as treatment for dementia. APA PsycNET. 2018: 615-629.

36. Norton MJ, Allen RS, Lynn Snow A, Michael Hardin J, Burgio LD. Predictors of need-driven behaviors in nursing home residents with dementia and associated certified nursing assistant burden. Aging Ment Health. 2010; 14: 303-309.

37. Lyketsos CG, Steele C, Galik E, Rosenblatt A, Steinberg M, Warren A, et al. Physical aggression in dementia patients and its relationship to depression. Am J Psychiatry. 1999; 156: 66-71.

38. Hall GR. Progressively lowered stress threshold; a conceptual model for care of adults with Alzheimer's disease. Arch Psychiatr Nurs. 1987; 1: 399-406.

39. Smith M, Gerdner LA, Hall GR, Buckwalter KC. History, development, and future of the progressively lowered stress threshold: A conceptual model for dementia care. J Am Geriatr Soc. 2004; 52: 1755-1760.

40. Gitlin LN, Winter L, Burke J, Chernett N, Dennis MP, Hauck WW. Tailored activities to manage neuropsychiatric behaviors in persons with dementia and reduce caregiver burden: $A$ randomized pilot study. Am J Geriatr Psychiatry. 2008; 16: 229-239.

41. de Oliveira AM, Radanovic M, Homem de Mello PC, Buchain PC, Dias Vizzotto A, Harder J, et al. An intervention to reduce neuropsychiatric symptoms and caregiver burden in dementia: $P$ reliminary results from a randomized trial of the tailored activity program-outpatient version. Int J Geriatr Psychiatry. 2019; 34: 1301-1307.

42. Galindo-Garre F, Volicer L, van der Steen JT. Factors related to rejection of care and behaviors directed towards others: A longitudinal study in nursing home residents with dementia. Dement Geriatr Cogn Dis Extra. 2015; 5: 123-134.

43. van der Ploeg ES, Eppingstall B, Camp CJ, Runci SJ, Taffe J, O'Connor DW. A randomized crossover trial to study the effect of personalized, one-to-one interaction using Montessoribased activities on agitation, affect, and engagement in nursing home residents with dementia. Int Psychogeriatr. 2013; 25: 565-575.

44. Lichtenberg PA, Murman DL, Mellow AM. Handbook of dementia: Psychological, neurological, and psychiatric perspectives. John Wiley \& Sons; 2004. 
45. Booth S, Zizzo G, Robertson J, Goodwin Smith I. Positive Interactive Engagement (PIE): A pilot qualitative case study evaluation of a person-centred dementia care programme based on Montessori principles. Dementia. 2018: 1471301218792144.

46. Judge KS, Menne HL, Whitlatch CJ. Stress process model for individuals with dementia. Gerontologist. 2010; 50: 294-302.

47. Pioli MF. Global and caregiving mastery as moderators in the caregiving stress process. Aging Ment Health. 2010; 14: 603-612.

48. Gitlin LN, Winter L, Dennis MP, Hodgson N, Hauck WW. Targeting and managing behavioral symptoms in individuals with dementia: A randomized trial of a nonpharmacological intervention. J Am Geriatr Soc. 2010; 58: 1465-1474.

49. Choi SSW, Budhathoki C, Gitlin LN. Impact of three dementia-related behaviors on caregiver depression: The role of rejection of care, aggression, and agitation. Int J Geriatr Psychiatry. 2019; 34: 966-973.

50. Farran CJ, Gilley DW, McCann JJ, Bienias JL, Lindeman DA, Evans DA. Efficacy of behavioral interventions for dementia caregivers. West J Nurs Res. 2007; 29: 944-960.

51. Judge KS, Yarry SJ, Looman WJ, Bass DM. Improved strain and psychosocial outcomes for caregivers of individuals with dementia: Findings from project ANSWERS. Gerontologist. 2013; 53: 280-292.

52. Krestar ML, Looman W, Powers S, Dawson N, Judge KS. Including individuals with memory impairment in the research process: The importance of scales and response categories used in surveys. J Empir Res Hum Res Ethics. 2012; 7: 70-79.

53. Dawson NT, Powers SM, Krestar M, Yarry SJ, Judge KS. Predictors of self-reported psychosocial outcomes in individuals with dementia. Gerontologist. 2013; 53: 748-759.

54. Powers SM, Dawson NT, Krestar ML, Yarry SJ, Judge KS. 'I wish they would remember that I forget:'The effects of memory loss on the lives of individuals with mild-to-moderate dementia. Dementia. 2016; 15: 1053-1067.

55. Menne HL, Judge KS, Whitlatch CJ. Predictors of quality of life for individuals with dementia: Implications for intervention. Dementia. 2009; 8: 543-560.

56. Park M, Choi S, Lee SJ, Kim SH, Kim J, Go Y, et al. The roles of unmet needs and formal support in the caregiving satisfaction and caregiving burden of family caregivers for persons with dementia. Int Psychogeriatr. 2018; 30: 557-567.

57. Davis LL, Buckwalter K, Burgio LD. Measuring problem behaviors in dementia: Developing a methodological agenda. ANS Adv Nurs Sci. 1997; 20: 40-55.

58. Cohen-Mansfield J, Werner P, Marx MS. An observational study of agitation in agitated nursing home residents. Int Psychogeriatr. 1989; 1: 153-165.

59. Cohen-Mansfield J. Conceptualization of agitation: Results based on the Cohen-Mansfield agitation inventory and the agitation behavior mapping instrument. Int Psychogeriatr. 1997; 8: 309-315.

60. Finkel SI, Lyons JS, Anderson RL. Reliability and validity of the Cohen-Mansfield agitation inventory in institutionalized elderly. Int J Geriatr Psychiatry. 1992; 7: 487-490.

61. Gustafsson C, Svanberg C, Müllersdorf M. Using a robotic cat in dementia care: A pilot study. J Gerontol Nurs. 2015; 41: 46-56.

62. Marin RS, Biedrzycki RC, Firinciogullari S. Reliability and validity of the Apathy Evaluation Scale. Psychiatry Res. 1991; 38: 143-162. 
63. Mahoney EK, Hurley AC, Volicer L, Bell M, Gianotis P, Hartshorn M, et al. Development and testing of the Resistiveness to Care Scale. Res Nurs Health. 1999; 22: 27-38.

64. Clarke DE, Reekum Rv, Simard M, Streiner DL, Freedman M, Conn D. Apathy in dementia: An examination of the psychometric properties of the apathy evaluation scale. J Neuropsychiatry Clin Neurosci. 2007; 19: 57-64.

65. Drachman DA, Swearer JM, O'Donnell BF, Mitchell AL, Maloon A. The caretaker obstreperousbehavior rating assessment (COBRA) scale. J Am Geriatr Soc. 1992; 40: 463-470.

66. Herz LR, Volicer L, Ross V, Rheaume Y. A single-case-study method for treating resistiveness in patients with Alzheimer's disease. Psychiatr Serv. 1992; 43: 720-724.

67. Volicer L. Palliative care in dementia. Prog Palliat Care. 2013; 21: 146-150.

68. Teri L, Truax P, Logsdon R, Uomoto J, Zarit S, Vitaliano PP. Assessment of behavioral problems in dementia: The revised memory and behavior problems checklist. Psychol Aging. 1992; 7: 622-631.

69. Cummings JL, Mega M, Gray K, Rosenberg-Thompson S, Carusi DA, Gornbein J. The Neuropsychiatric Inventory: Comprehensive assessment of psychopathology in dementia. Neurology. 1994; 44: 2308-2308.

70. Raglio A, Bellelli G, Traficante D, Gianotti M, Ubezio MC, Villani D, et al. Efficacy of music therapy in the treatment of behavioral and psychiatric symptoms of dementia. Alzheimer Dis Assoc Disord. 2008; 22: 158-162.

71. Reisberg B, Borenstein J, Salob SP, Ferris SH. Behavioral symptoms in Alzheimer's disease: Phenomenology and treatment. J Clin Psychiatry. 1987; 48: 9-15.

72. Reisberg B, Monteiro I, Torossian C, Auer S, Shulman MB, Ghimire S, et al. The BEHAVE-AD assessment system: A perspective, a commentary on new findings, and a historical review. Dement Geriatr Cogn Disord. 2014; 38: 89-146.

73. Hepburn KW, Lewis M, Sherman CW, Tornatore J. The savvy caregiver program: Developing and testing a transportable dementia family caregiver training program. Gerontologist. 2003; 43: 908-915.

74. Kiyak HA, Teri L, Borson S. Physical and functional health assessment in normal aging and in Alzheimer's disease: Self-reports vs family reports. Gerontologist. 1994; 34: 324-331.

75. La Rue A, Watson J, Plotkin DA. Retrospective accounts of dementia symptoms: Are they reliable? Gerontologist. 1992; 32: 240-245.

76. Drinka TJ, Smith JC, Drinka PJ. Correlates of depression and burden for informal caregivers of patients in a geriatrics referral clinic. J Am Geriatr Soc. 1987; 35: 522-525.

77. Hongisto K, Hallikainen I, Selander T, Törmälehto S, Väätäinen S, Martikainen J, et al. Quality of Life in relation to neuropsychiatric symptoms in Alzheimer's disease: 5-year prospective ALSOVA cohort study. Int J Geriatr Psychiatry. 2018; 33: 47-57.

78. Breland JY, Barrera TL, Snow AL, Sansgiry S, Stanley MA, Wilson N, et al. Correlates of pain intensity in community-dwelling individuals with mild to moderate dementia. Am J Alzheimers Dis Other Demen. 2015; 30: 320-325.

79. Judge KS, Camp CJ, Orsulic-Jeras S. Use of Montessori-based activities for clients with dementia in adult day care: Effects on engagement. Am J Alzheimers Dis. 2000; 15: 42-46.

80. Milev RV, Kellar T, McLean M, Mileva V, Luthra V, Thompson S, et al. Multisensory stimulation for elderly with dementia: A 24-week single-blind randomized controlled pilot study. Am J Alzheimers Dis Other Demen. 2008; 23: 372-376. 
81. McCurry SM, Gibbons LE, Logsdon RG, Vitiello MV, Teri L. Nighttime insomnia treatment and education for Alzheimer's disease: A randomized, controlled trial. J Am Geriatr Soc. 2005; 53: 793-802.

82. Cohen-Mansfield J, Marx MS, Rosenthal AS. Dementia and agitation in nursing home residents: How are they related? Psychol Aging. 1990; 5: 3-8.

83. Sposito G, Barbosa A, Figueiredo D, Yassuda MS, Marques A. Effects of multisensory and motor stimulation on the behavior of people with dementia. Dementia. 2017; 16: 344-359.

84. Sauer PE, Fopma-Loy J, Kinney JM, Lokon E. "It makes me feel like myself": Person-centered versus traditional visual arts activities for people with dementia. Dementia. 2016; 15: 895-912.

85. Zeisel J, Skrajner MJ, Zeisel EB, Wilson MN, Gage C. Scripted-IMPROV: Interactive improvisational drama with persons with dementia-effects on engagement, affect, depression, and quality of life. Am J Alzheimers Dis Other Demen. 2018; 33: 232-241.

86. Moyle W, Murfield J, Jones C, Beattie E, Draper B, Ownsworth T. Can lifelike baby dolls reduce symptoms of anxiety, agitation, or aggression for people with dementia in long-term care? Findings from a pilot randomised controlled trial. Aging Ment Health. 2019; 23: 1442-1450.

87. Luyten T, Braun S, Jamin G, van Hooren S, de Witte L. How nursing home residents with dementia respond to the interactive art installation 'VENSTER': A pilot study. Disabil Rehabil Assist Technol. 2018; 13: 87-94.

88. Orsulic-Jeras S, Judge KS, Camp CJ. Montessori-based activities for long-term care residents with advanced dementia: Effects on engagement and affect. Gerontologist. 2000; 40: 107-111.

89. Bourgeois MS, Schulz R, Burgio LD, Beach S. Skills training for spouses of patients with Alzheimer's disease: Outcomes of an intervention study. J Clin Geropsych. 2002; 8: 53-73.

90. Bass DM, Clark PA, Looman WJ, McCarthy CA, Eckert S. The Cleveland Alzheimer's managed care demonstration: Outcomes after 12 months of implementation. Gerontologist. 2003; 43: 73-85.

91. Judge KS, Bass DM, Snow AL, Wilson NL, Morgan R, Looman WJ, et al. Partners in dementia care: A care coordination intervention for individuals with dementia and their family caregivers. Gerontologist. 2011; 51: 261-272.

92. Maslow K. Translating innovation to impact: Evidence-based interventions to support people with Alzheimer's disease and their caregivers at home and in the community. Administration on Aging and Alliance for Aging Research. 2012.

93. Callahan CM, Boustani MA, Unverzagt FW, Austrom MG, Damush TM, Perkins AJ, et al. Effectiveness of collaborative care for older adults with Alzheimer disease in primary care: A randomized controlled trial. JAMA. 2006; 295: 2148-2157.

94. Burns R, Nichols LO, Martindale-Adams J, Graney MJ, Lummus A. Primary care interventions for dementia caregivers: 2-year outcomes from the REACH study. Gerontologist. 2003; 43: 547555.

95. Mittelman MS, Ferris SH, Shulman E, Steinberg G, Levin B. A family intervention to delay nursing home placement of patients with Alzheimer disease: A randomized controlled trial. JAMA. 1996; 276: 1725-1731.

96. Stanley MA, Calleo J, Bush AL, Wilson N, Snow AL, Kraus-Schuman C, et al. The Peaceful Mind program: A pilot test of a cognitive-behavioral therapy-based intervention for anxious patients with Dementia. Am J Geriatr Psychiatry. 2013; 21: 696-708. 
97. Gitlin LN, Hauck WW, Dennis MP, Winter L. Maintenance of effects of the home environmental skill-building program for family caregivers and individuals with Alzheimer's disease and related disorders. J Gerontol A Bio Sci Med Sci. 2005; 60: 368-374.

98. Alliance DA. Living with dementia: Changing the status quo white paper. 2016.

99. Gitlin LN, Maslow K, Khillan R. National research summit on care, services, and supports for persons with dementia and their caregivers. Report to the National Advisory Council on Alzheimer's Research, Care, and Services. 2018.

100. Orrell M, Hancock GA, Liyanage KCG, Woods B, Challis D, Hoe J. The needs of people with dementia in care homes: The perspectives of users, staff and family caregivers. Int Psychogeriatr. 2008; 20: 941-951.

101. Schölzel-Dorenbos CJ, Meeuwsen EJ, Olde Rikkert MG. Integrating unmet needs into dementia health-related quality of life research and care: Introduction of the hierarchy model of needs in dementia. Aging Ment Health. 2010; 14: 113-119.

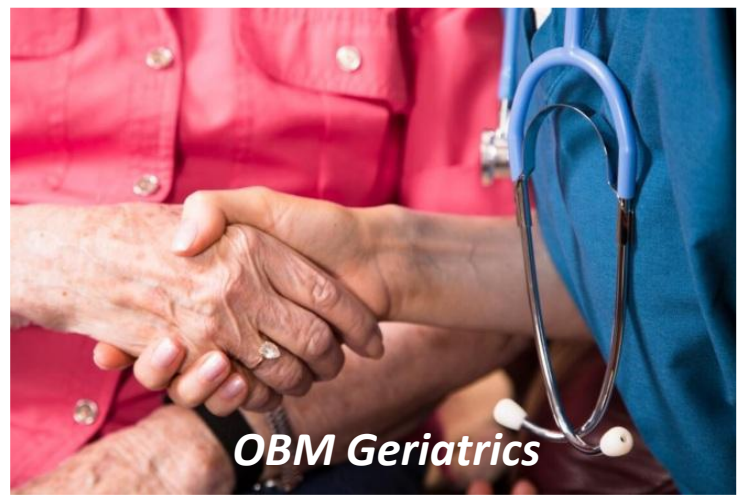

Enjoy $O B M$ Geriatrics by:

1. Submitting a manuscript

2. Joining in volunteer reviewer bank

3. Joining Editorial Board

4. Guest editing a special issue

For more details, please visit: http://www.lidsen.com/journals/geriatrics 